\title{
Evolución de los precios del petróleo
}

\author{
DANNY RAMÍREZ ${ }^{1}$ AND WILDER GUERRERO ${ }^{1}$ \\ ${ }^{1}$ Universidad Nacional Autónoma de Honduras , mail: danny.ramrez7@gmail.com , mail: hermogenes92@gmail.com
}

Recibido: 22 de Marzo de 2015 / Aceptado: 4 de Mayo de 2015

\begin{abstract}
Resumen
The price of oil and all the factors that determine its evolution have challenged any existing prediction models. This has generated the search for models, allowing as far as possible, a scientific analysis of financial markets. This has led to the development of a new area of investigation, which is econophysics, where certain physical models are very promising for scientific analysis of economic systems. Here, we develop a model that aims to predict the WTI oil price for the years 2014-2024, using as essencial variable, the historical volatility of prices for the years 1986-2013.
\end{abstract}

Keywords: Economy, predictability, oil.

El precio del petróleo, así como los factores que determinan su evolución, desafían cualquier modelo de predicción existente. Esto ha motivado la búsqueda de nuevos modelos, que permitan un análisis científico de los sistemas económicos, especialmente en el área de predictibilidad de los mercados financieros, impulsando, en cierta medida, el desarrollo de una nueva ciencia, llamada Econofísica; mediante la cual, se ha demostrado que ciertos modelos de la Física son potencialmente prometedores en el tratamiento científico de los sistemas económicos. Recientemente, la dinámica de los mercados financieros, ha llegado a ser de mucho interés para algunos físicos, debido a su amplio y complejo comportamiento de escalamiento, análogo a lo que comúnmente se observa en los sistemas físicos con muchas unidades que interactúan todo el tiempo. Así desde la perspectiva de la Econofísica, aquí se desarrolla un nuevo modelo para intentar predecir el precio del petróleo WTI, para el periodo 2014-2024, tomando como variable fundamental la volatilidad histórica del precio tabulado desde el año 1986 a octubre de 2013.

Palabras clave: Economía, predictibilidad, petróleo.

\section{INTRODUCCIÓN}

工 A energía juega un papel fundamental en el movimiento del mundo, y en la actualidad, la producción de ésta, es en extremo dependiente del petróleo y sus derivados. Dicho movimiento está, a su vez, directamente relacionado con la economía, generando una relación recíproca entre el precio del petróleo y la economía mundial.

Esta relación puede visualizarse en los siguientes ejemplos:

- El 80 - $90 \%$ de todo el transporte, funciona con productos petrolíferos.

- El 50 - $75 \%$ de todo el petróleo, se usa para el transporte.

- Todos los productos petroquímicos se hacen con petróleo.

- El $99 \%$ de todos los lubricantes se hacen con productos petrolíferos.

- El $95 \%$ de todos los bienes en las tiendas, llegan allí utilizando petróleo.
- El $99 \%$ de nuestra comida está relacionada con el petróleo o el gas para los fertilizantes, química, labrado y cultivo de la tierra y distribución.

- El petróleo es la fuente más importante de energía primaria del planeta y representa alrededor de un $35 \%$ del consumo energético mundial.

Una característica singular del precio del petróleo es su volatilidad. El precio del petróleo se rige a corto plazo más por factores externos a la estructura del mercado (la evolución de la cotización de las monedas con respecto al dólar, la variación de los niveles de inventarios, los conflictos políticos en las zonas productoras o cualquier fenómeno natural o social), que por la oferta y la demanda.

La volatilidad del precio del petróleo encierra el efecto tanto de esos factores externos, como de los factores estructurales del mercado. Así pues, analizar el comportamiento histórico de la volatilidad, significa analizar el efecto histórico de todos esos factores que afectan el mercado petrolero. Entonces cabe preguntarnos, ¿Es posible predecir el rango de volatilidad del precio del petróleo en los próximos años, desde la perspectiva de la Econofísica, tomando como base su comportamiento histórico? ¿Si eso es posible, entonces, es posible establecer escenarios 
probabilísticos para el precio del petróleo en los próximos años?

Existe una gama de factores externos a la estructura del mercado, que determinan la evolución del precio del crudo en mayor medida que la oferta y la demanda, y que no es posible predecir fuera del concepto de volatilidad histórica. En teoría, se dice que todos los fenómenos, de cualquier índole que afectaron la evolución del precio del petróleo en cierto periodo de tiempo, estan enmarcados dentro de su volatilidad histórica y por lo tanto, en éste modelo de predicción, se toma a ésta como variable fundamental.

\section{Fundamento TeÓRICO}

\section{A. Correlación}

La relación esperada entre el valor de una serie en el tiempo $t$ y sus valores en el tiempo $(t+\tau)$ es una medida de la correlación presente en una serie. Una serie de tiempo estacionaria tiene una correlación que sólo depende del periodo de tiempo $\tau$ entre las dos observaciones y el decrecimiento hasta cero, lo suficientemente rápido para que $\tau$ aumente, reflejando el hecho de que la influencia de los valores anteriores disminuye con los intervalos considerados. La velocidad de este decrecimiento es una medida de la "memoria" del proceso estocástico, que puede ser a largo o corto plazo.

\section{B. Autocorrelación}

La autocorrelación se define como la correlación existente entre los miembros de una serie de observaciones ordenadas en el tiempo o en el espacio, es decir, un periodo de crecimiento, en una serie de tiempo, es seguido de otro análogo. Una forma de medir el tipo de correlación existente en una serie de tiempo, es mediante el exponente de Hurst, $H$. Si $0.5<H<1$, se dice que existe autocorrelación o persistencia a largo plazo. Para el caso en que el exponente de Hurst esté entre $0<H<0.5$ tendremos una autocorrelación negativa, es decir, que el aumento tiende a ser seguido por una disminución.

\section{Exponente de Hurst}

El exponente de Hurst es una medida de independencia de las series de tiempo que fue estudiada inicialmente por el científico británico Harold Edwin Hurst(1880-1978), como elemento para distinguir series fractales. Hurst descubrió que muchos fenómenos naturales exhiben un comportamiento que puede ser caracterizado por un proceso aleatorio sesgado, en el cual existe "memoria de largo plazo" entre las observaciones, es decir, que los eventos de un período influyen en todos los siguientes.

Posteriormente, las ideas de Hurst fueron retomadas por Benoit B. Mandelbrot quien generalizó su trabajo y lo llamó análisis de rango reescalado $(R / S)$, definido como un método estadístico utilizado para evaluar la ocurrencia de eventos poco comunes, dando origen a una herramienta ideal para procesos físicos y financieros, aunque no se limita solamente a este tipo de eventos, por lo que puede ser usado en cualquier serie de tiempo.

La estimación del exponente de Hurst, $H$, fue desarrollada originalmente en la hidrología. Sin embargo, las modernas técnicas para estimar el exponente de Hurst vienen de la teoría de fractales. Éste muestra el grado de rugosidad de las series de tiempo y dado que los valores de $H$ van de 0 a 1 ; indica si la serie es aleatoria $(H \sim 0.5)$, antipersistente $(H<0.5)$ o persistente $(0.5<H<1)$.

El análisis $(R / S)$ consiste en escoger un cierto número de escalas, obtener la media del vector, calcular las diferencias de cada valor con respecto a la media y hacer una suma acumulativa de ellas. El rango es la diferencia entre el valor máximo y el valor mínimo de esta suma. $\mathrm{Al}$ dividir entre la desviación estandar se obtiene $(R / S)$, la cual, según Hurst, es proporcional a $c t^{H}$, donde $c$ es una constante, (0.5), y $t$ es la escala en cuestión. Asi que al graficar $\log (R / S)$ vs $t, H$ es la pendiente de la recta.

\section{Dimensión Fractal}

La dimensión fractal se puede definir como el número que sirve para cuantificar el grado de irregularidad y fragmentación de un conjunto geométrico o de un objeto natural (Strecker, 2004).

El exponente de Hurst puede ser determinado a partir de la dimensión fractal o viceversa, utilizando la fórmula:

$$
D=2-H
$$

\section{E. Análisis de Rango Reescalado $(R / S)$}

El análisis de rango reescalado permite encontrar el exponente de Hurst, el cual es un valor numérico que hace posible determinar la autocorrelación en una serie de tiempo.

A continuación se describe la metodología desarrollada por Hurst: Se inicia con una serie de tiempo de tamaño $M$, pero como interesan los rendimientos logarítmicos entonces la serie original se reduce a una nueva serie de tiempo de tamaño $N=M$, donde cada rendimiento está definido por:

$$
N_{i}=\log \left(\frac{M_{i+1}}{M_{i}}\right), \quad i=1,2,3, \ldots, N
$$

Este período de tiempo $N$ se divide en $A$ subperíodos contiguos de longitud $n$, tal que $A n=N$. Se nombra cada uno de los subperíodos o subgrupos $I_{a}$, con $a=$ $1,2,3, \ldots, A$. Cada elemento en $I_{a}$, es etiquetado $N_{k a}$, 
tal que $k=1,2,3, \ldots, n$. Para cada subperíodo $I_{a}$ de longitud $n$, el valor promedio está definido por la siguiente expresión:

$$
e_{a}=\frac{1}{N} \sum_{k=1}^{n} N_{k, a}
$$

Las diferencias de cada elemento $N_{k, a}$ con respecto a la media $e_{a}$ para cada subperiodo $I_{a}$ se van sumando para obtener la serie de tiempo acumulada $\left(X_{k, a}\right)$, definida como:

$$
X_{k, a}=\sum_{i=1}^{k}\left(N_{i, a}-e_{a}\right), k=1,2,3, \ldots, n .
$$

Se calcula la desviación estándar muestral $S I_{a}$ de la forma tradicional para cada periodo $I_{a}$ :

$$
S I_{a}=\left(\frac{1}{n} \sum_{i=1}^{k}\left(N_{i, a}-e_{a}\right)^{2}\right)^{\frac{1}{2}}
$$

Donde $k=1,2, \cdots, n$

El rango $R I_{a}$ se define como la diferencia entre el valor máximo y el valor mínimo de $X_{k, a}$ para cada subperiodo $I_{a}$ :

$$
R I_{a}=\operatorname{Max}\left(X_{k, a}\right)-\operatorname{Min}\left(X_{k, a}\right),
$$

con $1 \leq k \leq n$

Para cada período $I_{a}$, su rango $R I_{a}$ se normaliza dividiendo por su desviación estándar muestral $S I_{a}$ correspondiente. Por lo tanto el rango reescalado para cada subperiodo $I_{a}$ es igual a $(R I-a) / S I_{a}$. Como tenemos periodos continuos de longitud, entonces tomamos el valor promedio $(R / S)$ para períodos de longitud y que está definido como:

$$
(R / S)_{n}=\frac{1}{A} \sum_{a=1}^{A} \frac{R I_{a}}{S I_{a}}
$$

La longitud $n$ o el tamaño del subperíodo se incrementa al siguiente valor posible de tal forma que $M-1$ sean un valor entero. Iniciamos con el valor más pequeño de acuerdo a la condición anterior y se repite el proceso hasta $n=(M-1) / 2$ utilizando siempre la serie completa. Posteriormente aplicamos una regresión de mínimos cuadrados de $\log (R / S)_{n}$ contra $\log (n)$. La ordenada al origen es el $\log (c)$ y la pendiente de la ecuación es la estimación del exponente de Hurst, $H$. (Recuérdece que, según Hurst, $R / S \sim c t^{H}$ y al linealizar esa ecuación, tenemos que: $\log (R / S) \simeq H \log (t)+\log (c)$ ).

\section{F. Volatilidad}

La Volatilidad, trata de cuantificar la variabilidad o dispersión de un activo en un horizonte de tiempo dado, y aunque el concepto de volatilidad, desde un punto de vista estadístico, está relacionado con el de desviación estándar, no existe una definición apodíctica y universalmente aceptada para ésta. Para el precio del petróleo en un periodo de tiempo, de $t$ a $t+\tau$, se ha definido como el valor absoluto del rendimiento logarítmico del precio: $\delta=\log \left(\frac{P_{t+\tau}}{P_{t}}\right)$, donde $P_{t+\tau}$ es el precio en el tiempo $t+\tau$ y $P_{t}$ es el precio en el tiempo $t$.

Esta definición no es directamente útil para el desarrollo de este modelo, porque sólo toma en cuenta los valores extremos de dicho horizonte de tiempo, pero partimos de ella para definir una nueva ecuación para la volatilidad. Así, encontramos la siguiente relación:

$$
\delta=\log \left(\frac{P_{t+\tau}}{P_{t}}\right)
$$

, entonces

$$
\delta \approx\left(\frac{\Delta P}{\bar{P}}\right)^{2} \frac{\exp \left(\frac{\Delta P}{\bar{P}}\right)}{\exp \left(\frac{\Delta P}{\bar{P}}\right)-1}
$$

donde $\Delta P=\left(P_{t+\tau}-P_{t}\right) \quad$ y $\quad \bar{P}$ es el promedio.

Tomamos la aproximación de la ecuación (7) y sustituimos $\Delta P$ por la desviación estándar $\sigma$, la cual, al igual que $\bar{P}$, toma todo los valores de la muestra. Así redefinimos la ecuación de la volatilidad como sigue:

$$
\delta=\left(\frac{\sigma}{\bar{P}}\right)^{2} \frac{\exp \left(\frac{\sigma}{\bar{P}}\right)}{\exp \left(\frac{\sigma}{\bar{P}}\right)-1}
$$

\section{G. Ecuaciones Para Generar Los Tres Escena- rios Probabilísticos}

Bajo el supuesto, que la volatilidad encierra el efecto de todos los factores que alteran el mercado, nuestras ecuaciones de predicción fueron extraídas de la ecuación de dilatación líneal

$$
L=L_{0}+L_{0} \alpha \Delta T
$$

donde $\alpha$, es el coeficiente de dilatación líneal del material y $\Delta T$, el diferencial de temperatura. Así, para nuestras ecuaciones de predicción sólo cambiamos la longitud por el precio y en lugar de $\alpha$ y $\Delta T$, la dependencia es directamente de la volatilidad histórica.

Usamos la ecuacuión

$$
P_{k+}=P_{b a s e, j}+P_{b a s e, j} V_{2 i-1}
$$

para determinar el primer elemento del escenario a la alta. Donde $V_{2 i-1}$, representa los elementos impares de la serie de la volatilidad histórica. 
Y la ecuación

$$
P_{k-}=P_{b a s e, j}-P_{b a s e, j} V_{2 i}
$$

para determinar el primer elemento del escenario a la baja. Donde $V_{2 i}$, representa los elementos pares de la serie de la volatilidad histórica.

De esta manera podemos calcular el nuevo precio base:

$$
P_{\text {base }}=\frac{P_{(+)}+P_{(-)}}{2}
$$

\section{AnÁlisis del Precio Histórico}

- Tomamos un conjunto de 7014 valores del precio de cierre diario del crudo WTI, para el periodo enero de 1986 a octubre de 2013.

- Calculamos el exponente de Hurst de toda la muestra de datos tomando subperíodos desde 15 hasta 50 valores y vemos para qué periodo entre $15 \mathrm{y}$ 50, existe mayor persistencia. (Se eligió ese rango, porque para rangos mayores la serie de tiempo no mostraba persistencia.)

- Definimos el subperíodo $\tau$ para el cual existe mayor persistencia, calculamos la volatidad usando la ecuación (8) para cada subperíodo $\tau$, desde 1 hasta 7014 sumando 1 a cada extremo de éste, de manera que permanezca constante hasta cubrir toda la masa de datos.

- Luego que obtuvimos la serie de $7014-\tau$ valores, para la volatilidad, elegimos el último precio de nuestra serie de tiempo y lo tomamos como precio base.

A continuación se presentan algunas gráficas del comportamiento histórico del precio del crudo y también aquellas que resultaron de analizar si muestra o no persistencia a largo plazo.

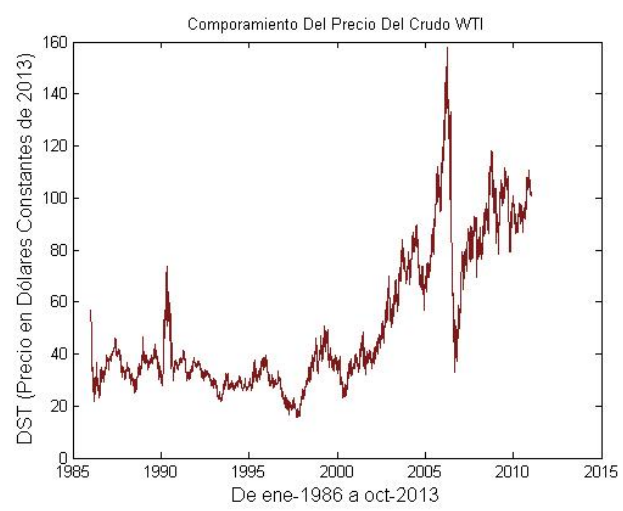

Figura 1: Comportamiento del precio del crudo WTI

En la Figura 1 puede verse la evolución del crudo WTI para el periodo (Enero de 1986 - Octubre de 2013)

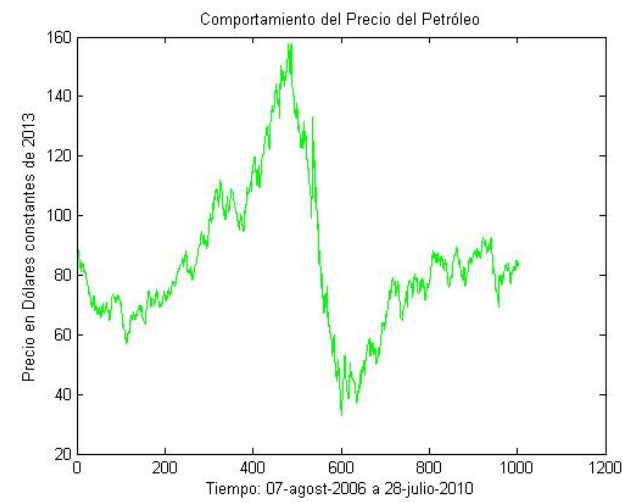

Figura 2: Comportamiento del precio del petróleo

En la Figura 2 se tienen los precios para el crudo WTI, donde puede verse un comportamiento bastante irregular que tuvo lugar en el año 2008. Tras 20 años con el precio del crudo casi siempre por debajo de los 25 dólares el barril, en 2003 comenzó una escalada que culminó con un máximo histórico de 147 dólares para luego caer hasta los 40 dólares en diciembre del mismo año. Este periodo se conoce ya como la tercera crisis del petróleo.

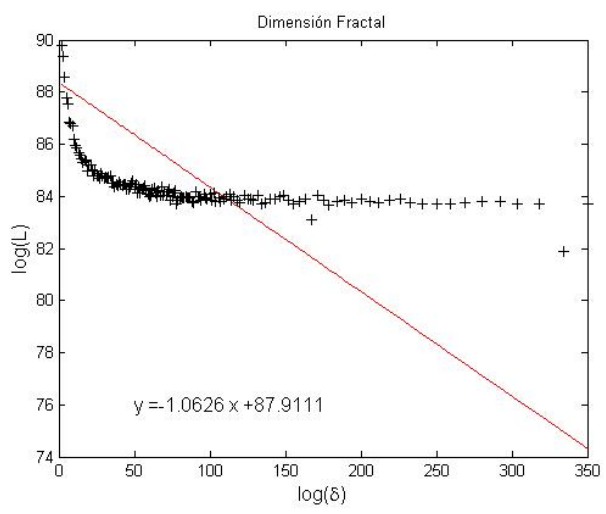

Figura 3: Dimensión Fractal

Se muestra en la figura 3 que la serie de tiempo del precio, aunque es persistente, no tiene un comportamiento fractal, porque el conjunto de puntos no se ajusta a la recta. Y por lo cual, la ecuación $D=2-H$, no se cumple, y fue necesario el análisis de rango reescalado, para calcular el exponente de Hurst. 


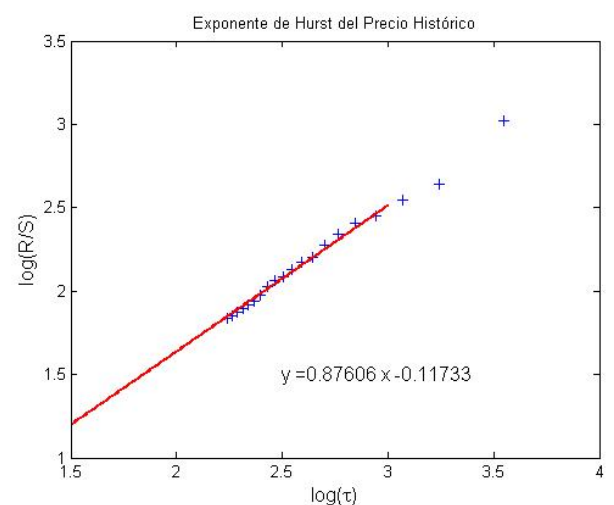

Figura 4: Exponente del Hurst precio histórico

En la Figura 4 se muestra el exponente de Hurst cuyo valor es $(H \approx 0.88)$ Esto nos muestra que el mercado petrolero es un proceso estocástico persistente con efectos de memoria a largo plazo. (existe invarianza de escala asociada a correlaciones positivas a largo plazo).

\section{Volatilidad Histórica}

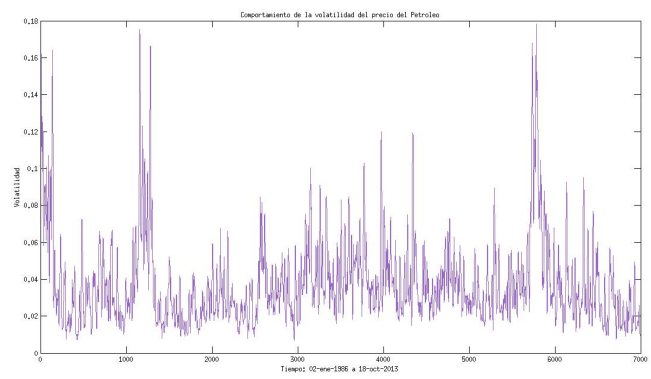

Figura 5: Comportamiento de la volatilidad del precio del crudo, para el periodo Enero de 1986 - Octubre de 2013

En la última década se han aplicado conceptos de la mecánica estadística tales como escalamiento, fractales, distribuciones de ley de potencia, autocorrelación y renormalización de grupos al análisis de los sistemas económicos y mercados financieros. Ello ha dado como resultado conclusiones contrarias a la creencia de que las fluctuaciones de precios son totalmente aleatorias.

Se muestra en la Figura 5 la volatilidad de los precios del petróleo. Los datos de esta gráfica fueron utilizados para la predicción de precios del petróleo, proyectando valores anuales desde 2014 hasta 2024.

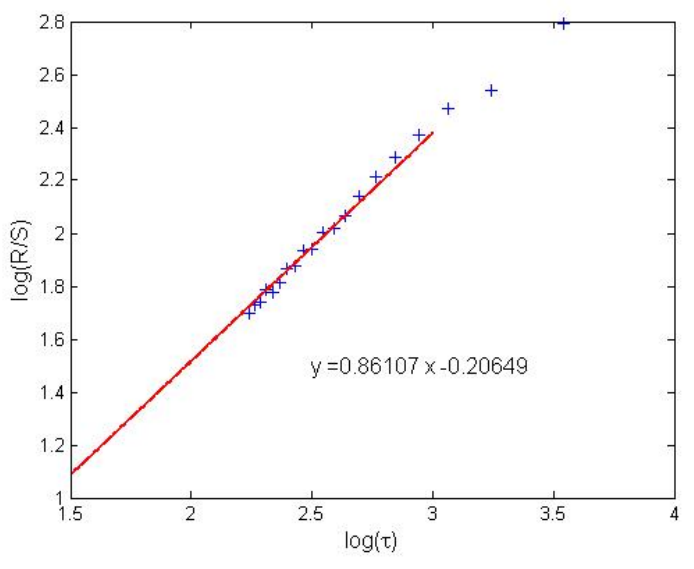

Figura 6: Exponente de Hurst de la Volatilidad

Podemos ver la figura 6, donde la pendiente de la recta representa el exponente de Hurst para la volatilidad cuyo valor es $H \approx 0.87$. Esto representa una autocorrelación positiva, que representa un aumento en termino de probabilidades para proyecciones de los precios WTI.

\section{Proyección del Precio Del CRUdo WTI}

De la ecuación 10 tenemos:

$$
P_{k+}=P_{b a s e, j}+P_{b a s e, j} V_{2 i-1}
$$

Y de la ecuación (11) :

$$
P_{k-}=P_{b a s e, j}-P_{b a s e, j} V_{2 i}
$$

Donde los subíndices $i, j, k$ varían según la proyección de la volatilidad y $k=j$. así, el nuevo precio estimado será:

$$
P_{b a s e, j+1}=\frac{P_{(k+)}+P_{(k-)}}{2}
$$

Con este nuevo precio base calculamos el segundo elemento de los escenarios extremos. Repetimos el ciclo anterior hasta que

$$
i=(7014-\tau) / 2
$$

En las ecuaciones (13) y (14) se usan las variables $V_{2 i-1}$ y $V_{2 i}$ las cuales representan los valores pares e impares de la serie calculada para la volatilidad, lo que, al igual que para los precios proyectados, se hizo con un programa elaborado en MATLAB . 


\section{Resultados}

Encontramos que:

- Para subperíodos de 20 días, $H \approx 0.88$, existe persistencia en la serie de tiempo del precio tabulado del petróleo WTI, por lo cual se tomó ese periodo como horizonte de tiempo, para calcular la serie de volatilidad histórica.

- Nuestra serie de tiempo de la volatilidad histórica, tambien es persistente para horizontes de 20 días.

- los tres escenarios probabilísticos proyectados, para horizontes de 20 días, tienen un comportamiento como se muestra en las gráfica siguientes.

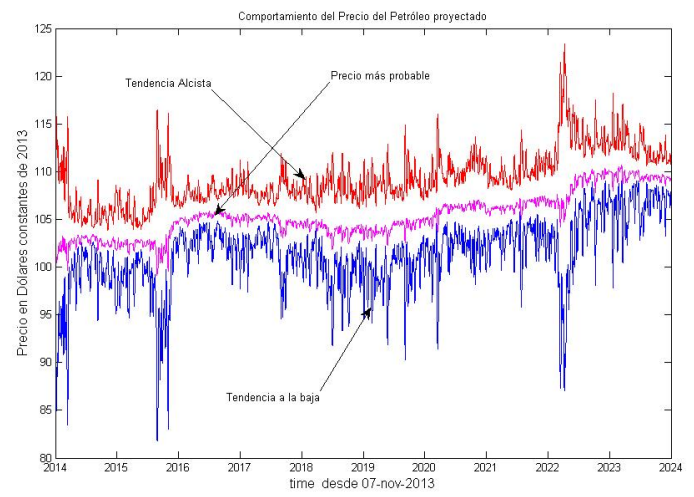

Figura 7: Escenarios probables

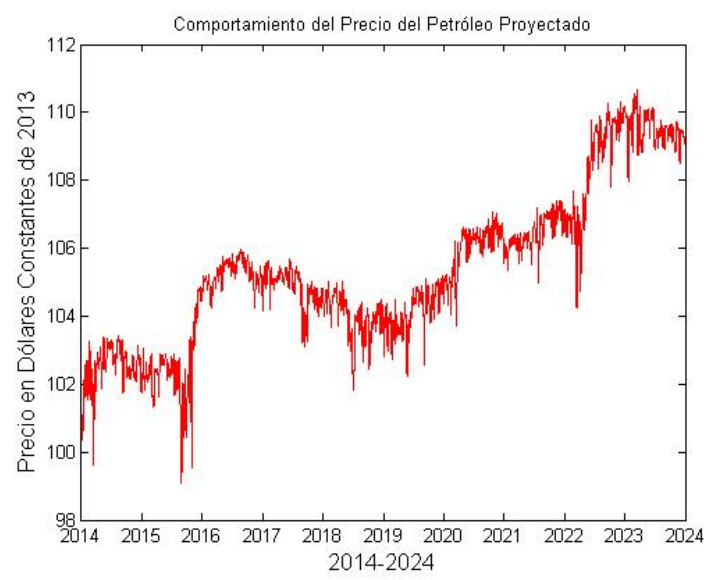

Figura 8: Comportamiento del precio del petróleo proyectado

En las Figuras 7 y 8 se muestran los resultados finales de de este trabajo. En la Figura 8 se expone el escenario más probable para los precios diarios entre los años 2014 al 2024.

En el Cuadro 1 pueden verse los valores promedios anuales en dólares constantes del 2013 correspondientes a este mismo intervalo de tiempo.

\begin{tabular}{|c|c|c|c|}
\hline Año & P. Alta & P. Baja & P. Probable \\
\hline 2014 & 106.81 & 98.14 & 102.48 \\
\hline 2015 & 107.13 & 98.20 & 102.67 \\
\hline 2016 & 108.06 & 98.22 & 105.27 \\
\hline 2017 & 107.99 & 101.50 & 104.75 \\
\hline 2018 & 108.29 & 99.34 & 103.82 \\
\hline 2019 & 109.10 & 99.88 & 104.49 \\
\hline 2020 & 110.06 & 102.45 & 106.26 \\
\hline 2021 & 111.68 & 101.57 & 106.62 \\
\hline 2022 & 113.28 & 105.93 & 109.61 \\
\hline 2023 & 111.96 & 106.83 & 109.39 \\
\hline 2024 & 112.95 & 107.81 & 110.38 \\
\hline
\end{tabular}

Cuadro 1: Precios proyectados en dólares constantes, 2013

\section{Discusión}

- Nuestros resultados provienen de establecer límites históricos en la volatilidad, por lo que esperamos que en condiciones estables, el comportamiento real del precio promedio anual se ajuste a nuestro escenario más probable.

- La ecuación (8) desafía a las ecuaciones ya establecidas para la determinación de la volatilidad, porque toma en cuenta todos los valores del horizonte de tiempo y arroja resultados, que siempre son positivos y adimensionales.

\section{REFERENCIAS}

[1] Alexander S Balankin y col. "Crossover from antipersistent to persistent behavior in time series possessing the generalyzed dynamic scaling law". En: Physical Review E 69.3 (2004), pág. 036121.

[2] Jan W Dash. Quantitative finance and risk management: a physicist's approach. World Scientific Pub., 2004.

[3] Edgar Israel García Otamendi. "Dinámica De Sistemas Complejos:. ${ }^{\mathrm{A}}$ nálisis Fractal Del Índice De Precios, Cotizaciones". Tesis doct. 2008.

[4] James D Hamilton. Understanding crude oil prices. Inf. téc. National Bureau of Economic Research, 2008.

[5] Bàrbara Llacay y Gilbert Peffer. Simulación realista de los mercados financieros con sistemas multiagentes. Inf. téc. Working Paper, 2008.

[6] Rosario N Mantegna y H Eugene Stanley. Introduction to econophysics: correlations and complexity in finance. Cambridge university press, 2000.

[7] Oswaldo Morales Matamoros, Alexander Balankin y Luis M Hernández Simón. "Metodología de predicción de precios del petróleo basada en dinámica fractal”. En: Científica 9.1 (2005), págs. 3-11. 
[8] Joseph L McCauley. Dynamics of markets: econophysics and finance. Vol. 31. Cambridge University Press Cambridge, 2004.

[9] Oswaldo Morales-Matamoros, Ricardo Tejeida-Padilla e Isaias Badillo-Piña. "Fractal behaviour of complex systems". En: Systems Research and Behavioral Science 27.1 (2010), págs. 71-86.

[10] Adauto Israel Ortiz Romero. "Construcción Y Programación De Un Algoritmo Paralelo Para Determnar El Exponente De Hurst". Tesis doct. 2009.

[11] Wolfgang Paul y Jörg Baschnagel. Stochastic processes: from physics to finance. Springer, 2000.

[12] Pierre Perron. "The great crash, the oil price shock, and the unit root hypothesis". En: Econometrica: Journal of the Econometric Society (1989), págs. 1361-1401.

[13] Didier Sornette, Ryan Woodard y Wei-Xing Zhou. "The 2006-2008 oil bubble: Evidence of speculation, and prediction". En: Physica A: Statistical Mechanics and its Applications 388.8 (2009), págs. 1571-1576.
[14] H Eugene Stanley y col. "Anomalous fluctuations in the dynamics of complex systems: from DNA and physiology to econophysics". En: Physica A: Statistical Mechanics and its Applications 224.1 (1996), págs. 302-321.

[15] H Eugene Stanley y col. "Econophysics: Can physicists contribute to the science of economics?" En: Physica A: Statistical Mechanics and its Applications 269.1 (1999), págs. 156-169.

[16] Johannes Voit. The statistical mechanics of financial markets. Vol. 2. Springer Berlin, 2003.

[17] Yudong Wang y Li Liu. "Is WTI crude oil market becoming weakly efficient over time?: New evidence from multiscale analysis based on detrended fluctuation analysis". En: Energy Economics 32.5 (2010), págs. 987-992.

[18] Bram leo Willems. "Aplicación de modelos físicos a la economía: una breve introducción a la econofísica". En: Sinergia e Innovación 1.09 (2013). 